\title{
Contribution to the ethnobotanical inventory of medicinal plants used for the treatment of typhoid fever in Adamaoua region, Cameroon
}

\author{
Simeon Pierre FODOUOP CHEGAING ${ }^{1,2^{*}}$, Didiane YEMELE MEFOKOU ${ }^{1,2}$, \\ Benjamin TALOM TANGUE ${ }^{1,2}$, Jean Baptiste SOKOUDJOU ${ }^{1}$, Serge TELEM- \\ GONE MENOUDJI ${ }^{2}$, Gabriel TCHUENTE KAMSU ${ }^{1}$ and Donatien GATSING ${ }^{1}$ \\ ${ }^{1}$ Research Unit of Microbiology and Antimicrobial Substances, Department of Biochemistry, University of \\ Dschang P.O. Box 67, Dschang, Cameroon. \\ ${ }^{2}$ Department of Biomedical Sciences, University of Ngaoundéré, PO. Box 454, Ngaoundéré, Cameroon. \\ ${ }^{*}$ Corresponding author; E-mail: saafodouop@yahoo.fr; Tel. +237696907805 /+237675723760
}

\author{
Received: 03-10-2020 Accepted: 27-12-2020 Published: 31-12-2020
}

\begin{abstract}
Since ancient Greek-Roman times, the use of plants to cure many human diseases is still common. The present ethnobotanical survey was conducted to contribute to the knowledge of medicinal plants used for the treatment of typhoid fever in three sub divisions of Vina division, Adamawa Cameroon. After having explained the importance of this study to interviewees, 41 traditional healers have agreed and delivered information regarding the medicinal plants they use as well as the different preparation and administration through a wellstructured questionnaire that was given to them on this matter. Among 41 traditional healers whose attended this study, 32 were men and 09 were women. The ethnobotanical survey allowed the identification of 70 plants belonging to 38 families. With a frequency of 11/70, the Fabaceae family was the most represented followed by that of Rubiaceae and Asteraceae (04/70 each). The leaves are the most used parts $(34.28 \%)$ followed by leaves + roots $(14.28 \%)$ and the whole plant $(12.86 \%)$. The majority of the recipes consisted of four to six plants (34.66), and were prepared by decoction (50\%), with water as the main solvent $(87.80 \%) .41 .56 \%$ of typhoid preparations are administered twice daily for a duration of 14 days (46.77\%). This is the first report on antityphoid herbal remedies in Vina division-Adamawa Cameroon. It would therefore be judicious for our government and research institution to investigate on their therapeutic properties in order to develop ameliorated and efficient phytomedicines.
\end{abstract}

(c) 2020 International Formulae Group. All rights reserved.

Keywords: Ethnopharmacology, phytomedecine, salmonelloses, Vina division.

\section{INTRODUCTION}

Salmonelloses are one of the most important food borne diseases which affect human and animal. In human beings, three serotypes including Salmonella typhi ( $S$. typhi), Salmonella paratyphi A (S. paratyphi $A)$ and Salmonella paratyphi B (S. paratyphi B) are more pathogenic. These serotypes cause typhoid and paratyphoid fevers (Gatsing et al., 2010). Typhoid fever is a widespread disease in many tropical countries. An estimated 212 million cases and 129,000 deaths occur worldwide each year (Steele et al., 2016). The global estimate of invasive non-typhoidal salmonella is about 3.4 million cases and the most vulnerable groups (1.9 million cases and 380000 deaths) are children and young adults in sub-Saharan Africa (Njole et al., 2020). 
This food and water borne disease is contacted after consumption of infected raw or semi coocked meat. The clinical manifestations of typhoid fever are usually nonspecific, such as sustained fever with fatigue, headache, abdominal pain, vomiting, or anorexia. Various organs, including the liver, have been involved in the course of typhoid fever, resulting in a wide spectrum of presentations (Habte et al., 2018).

Despite the permanent progress in pharmaceutical companies, resistance to different drug classes (Mfopa et al., 2017; Etou et al., 2019) has become an increasingly important problem (Madhulika et al., 2004, Lakshmi et al., 2006) especially in Subsahelian Africa where the income of $3 / 4$ of the total population do not allow them to afford and/or achieve antibiotherapy. In addition, although three vaccines are currently recommended by the WHO to prevent typhoid: an injectable vaccine on the purified $\mathrm{Vi}$ antigen (aka ViPS vaccine), the live attenuated oral Ty21a vaccine in the first instance and the latest generation typhoid conjugate vaccine (TCV), there is to date no vaccine against paratyphoid fever (Agwu et al., 2009). Natural products and mostly medicinal plants are thus the corner stone of health care delivery for these populations.

In Cameroon, several studies have revealed the effectiveness of the use of medicinal plants in the treatment of typhoid fever (Gatsing et al., 2005; Fodouop et al., 2015; Kengni et al., 2016; Lunga et al., 2014a; Tala et al., 2018; Sokoudjou et al., 2018; Sokoudjou et al., 2019). Therefore, there is no longer any doubt about indigenous knowledge and the use of medicinal plants to treat typhoid fever in Cameroon. Since this knowledge is held by adults and fewer young people are interested in ethnomedecine, it is important that this knowledge is documented and preserved for future research on efficacy and safety as well as the identification of chemical compounds that could serve as a marker for the development of standardized bioantibiotics.

Thus the present work aimed at carrying out an inventory of medicinal plants used for the treatment of typhoid fever in three localities of the Adamaoua region (Mbe, Martap and Ngaoundere 3) in order to know whether there is a consensus on the indigenous use of medicinal plants from different localities.

\section{MATERIALS AND METHODS Study area}

The Vina division was created from the breakup, in 1983, of the old Adamaoua division into five new divisions: Djerem, Faro et Deo, Mayo-Banyo, Mbere and Vina. The Vina Division has eight subdivisions: Belel, Mbe, Nganha, Ngaoundéré $I^{\mathrm{er}}$, Ngaoundéré $\mathrm{II}^{\mathrm{e}}$, Ngaoundere $\mathrm{III}^{\mathrm{e}}$, Nyambaka, Martap. It covers an area of $17196 \mathrm{~km}^{2}$. In 2005, its total population was estimated at 156050 (9.1 inhab $/ \mathrm{km}^{2}$ ) (ASC, 2013).

The Adamaoua region is located between the $6^{\text {th }}$ and ${ }^{8 t h}$ degrees of north latitude and between the $11^{\text {th }}$ and $15^{\text {th }}$ degrees of east longitude (Lamy et al., 2018). It is made up of all the high plateaus which cross it from West to East (Figure. 1). Its median position between the southern and northern parts of Cameroon makes this region a crossing point between the forest and the savannah, at the same time as it is a watershed. She was rightly baptized "Cameroon water tower".

As for the climate, it is of SudanoGuinean type with 02 seasons: a rainy season going from April to October and a dry season from November to March. The annual rainfall from 1600 to $1800(\mathrm{~mm})$ is spread over 7 to 8 months (Lamy et al., 2018). The soil, mainly consists of red ferralitic structures developed on old basalts. Various types of vegetation are also observed there. They range from meadows to shrub savannas to tree savannas dominated by Daniellia oliveri and Lophira lanceolata. The density of these characteristic species is clearly decreasing due to anthropogenic action (Mapongmetsem et al., 2012). Concerning the populations, they are composed of several ethnolinguistic groups of unequal distribution, the most representative of which are: Dii or Dourou, Gbaya, Haoussa, Mbororo, Mboum and Peulh (Mapongmetsem et al., 2009; Tchobsala and Mbolo, 2013). The study area includes three subdivisions of the Vina division namely: Mbe, Martap and Ngaoundéré III ${ }^{\mathrm{e}}$. 


\section{Data collection}

An ethnobotanical survey took place from June to November 2017 as previously described by Yemele et al. (2014), and it included forty one traditional healers aged between 15 and above 75 years. They were interviewed according to a questionnaire which was previously drawn up and comprised: the types of medication (medicinal plants or pharmaceutical products) used whenever sick; and for each medicinal plant used its medical use, part used during the preparation, mode of preparation, route of administration, the solvent used, the dosage, the duration of treatment and consumer's degree of satisfaction relatively to each treatment were noted. Interviews were conducted on the field during collection trips and after examination and seeking oral consent from informants, fresh plant specimens were collected, dried and stored in the laboratory. They were later identified at the Cameroon National Herbarium Yaoundé where their full scientific names and voucher number were obtained.

\section{Data analysis}

Descriptive statistic was used in this study. First of all, the information about the popular uses of the species collected, along with botanical information, was compiled into a database. The species were listed in alphabetical order by family, scientific name and frequency of use. The frequency of citation (FC) of the used plant species was evaluated using the following formula: FC (number of times a particular species was mentioned/total number of times that all species were mentioned).

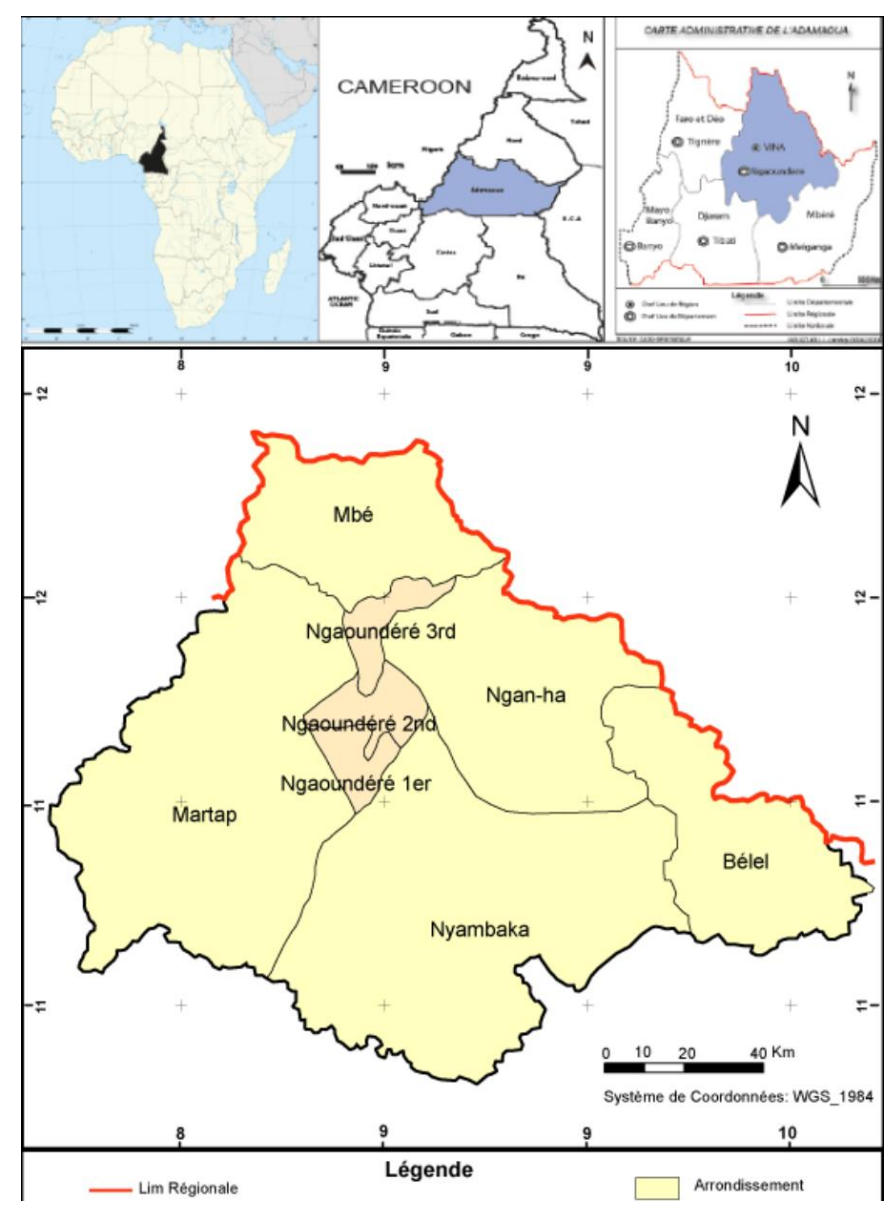

Figure 1: Study area (from the PSFE, 2010 database). 


\section{RESULTS}

\section{Demographic characteristics of informants}

Among the 41 traditional healers interviewed during this study, 32 were men and 9 women (Table 1). This table also shows a fair distribution of age-related surveys. With a frequency of 16/41, Mbe town had the highest numbers of traditional healers who participated in the inquiry (Table 1).

\section{Different plant species recorded and origin of the knowledge}

70 plants belonging to 38 families were identified (Table 2). With a frequency of 11/70, Fabaceae was the most represented family followed by that of Rubiaceae and Asteraceae (04/70 each). Persea americana (20/308) Annona senegalensis 17/308; Carica papaya 17/308; Psidium guayava (17/308) Citrus limon (11/308): Mangifera indica (14/308); Piliostigma thonningii (11/308) and Harungana madagascariensis (11/308) were the most cited plant (Table 2).

Regardless of the study district, the practice of traditional medicine is mostly inherited by offspring from their ancestors. However, many people practise it following a long period of learning from traditional healers (Table 3).

\section{Parts of plant used, mode of preparation of different recipes and mode of administration}

The most plant parts used were leaves (34.28\%), followed by leaves+stem bark $(14.28 \%)$ and whole plant $(12.86 \%)$ (Figure . 3; Table 2).

Among these, decoction is the most used $(56 \%)$ followed by maceration $(32.93 \%)$. With a percentage of 87.5 , water was the main solvent of extraction (Figure 5; Table 3). Other solvents were oil (4.88\%), honey (3.66\%), cow milk, (3.66\%). All these preparations were administered orally, and the duration of treatment range from four to fourteen days (Table 3). As far as doses in concerned, one glass of the preparation was taken two $(41.56 \%)$ and tree $(38.96 \%)$ time per day (Figure 6; Table 3).

Among the 41 recipes obtained, $15.58 \%$ were made by using one plant, $33.76 \%$ with a mixture of two to three plants, $37.66 \%$ with four to six plants and $13 \%$ with seven to eleven plants (Figure 2). Apart from typhoid fever, these plant are used to cure several other complaints (Table 2).

Table 1: Distribution of informants according to their age, sex and subdivision.

\begin{tabular}{|c|c|c|c|c|}
\hline \multirow{2}{*}{ Age } & \multirow{2}{*}{ subdivision } & \multicolumn{2}{|c|}{ sex } & \multirow[t]{2}{*}{ Total } \\
\hline & & Male & Female & \\
\hline \multirow{3}{*}{$15-30$} & Martap & 02 & 01 & \multirow{3}{*}{09} \\
\hline & Mbe & 01 & // & \\
\hline & Ngaoundere 3 & 05 & // & \\
\hline \multirow{3}{*}{$30-45$} & Martap & 01 & 01 & \multirow{3}{*}{08} \\
\hline & Mbe & 02 & 01 & \\
\hline & Ngaoundere 3 & // & 03 & \\
\hline \multirow{3}{*}{$45-60$} & Martap & 02 & $/ /$ & \multirow{3}{*}{10} \\
\hline & Mbe & 07 & 01 & \\
\hline & Ngaoundere 3 & // & // & \\
\hline \multirow{3}{*}{$65-75$} & Martap & 06 & $/ /$ & \multirow{3}{*}{09} \\
\hline & Mbe & 01 & 01 & \\
\hline & Ngaoundere 3 & 01 & // & \\
\hline \multirow{3}{*}{75 -above } & Martap & 01 & // & \multirow{3}{*}{05} \\
\hline & Mbe & 02 & // & \\
\hline & Ngaoundere 3 & 01 & 01 & \\
\hline & Total & 32 & 09 & 41 \\
\hline
\end{tabular}


Table 2: Classification of plants counted according to their family, frequency of citation and other uses.

\begin{tabular}{|c|c|c|c|c|c|}
\hline Family & Scientific name & $\begin{array}{l}\text { Part } \\
\text { used }\end{array}$ & $\begin{array}{l}\text { Mode of } \\
\text { prepa- } \\
\text { ration } \\
\text { (solvent) }\end{array}$ & FC & Other uses \\
\hline Aloeaceae & Aloe vera & $\mathrm{L}$ & $\mathrm{M}(\mathrm{W})$ & $03 / 308$ & $\begin{array}{l}\text { skin infections, gastritis, laxative, } \\
\text { abdominal pain }\end{array}$ \\
\hline \multirow[t]{2}{*}{ Anacardiaceae } & Lannea acida & $\mathrm{L}, \mathrm{SB}$ & $\mathrm{D}(\mathrm{W})$ & $07 / 308$ & Yellow fever \\
\hline & Mangifera indica $L$ & $\mathrm{~L}, \mathrm{SB}$ & $\mathrm{D}(\mathrm{W})$ & $14 / 308$ & $\begin{array}{l}\text { Jaundice, Stomach ache, Itching, } \\
\text { Gastric reflux, Malaria, Yellow } \\
\text { fever, Hepatitis, diarrhea, } \\
\text { Respiratory pain, }\end{array}$ \\
\hline \multirow[t]{2}{*}{ Annonaceae } & $\begin{array}{l}\text { Annonas } \\
\text { senegalensis }\end{array}$ & $\mathrm{L}, \mathrm{SB}$ & $\mathrm{B}(\mathrm{W})$ & $17 / 308$ & $\begin{array}{l}\text { diarrhea, vermifuge, diabetes, } \\
\text { osteoarthritis, anemia, epilepsy, } \\
\text { dysentery, malaria, pneumonia }\end{array}$ \\
\hline & Annona muricata & $\mathrm{L}$ & $\mathrm{M}, \mathrm{I}, \mathrm{D}(\mathrm{W})$ & $02 / 308$ & Stomach ache \\
\hline Apiaceae & Apium graveolens & WP & $\mathrm{D}(\mathrm{W})$ & $02 / 308$ & dysentery \\
\hline Apocynaceae & Voacanga africana & $\mathrm{L}, \mathrm{SB}$ & $\mathrm{D}(\mathrm{W})$ & $02 / 308$ & $\begin{array}{l}\text { anti helmetic, epilepsis, wound, } \\
\text { tooth decay }\end{array}$ \\
\hline \multirow[t]{4}{*}{ Asteraceae } & Bidens pilosalinn & WP & $\mathrm{M}, \mathrm{D}(\mathrm{W})$ & $05 / 308$ & Jaundice, dysentery \\
\hline & $\begin{array}{l}\text { Crhysanthellum } \\
\text { africanium }\end{array}$ & WP & $\mathrm{M}, \mathrm{D}(\mathrm{W})$ & $04 / 308$ & Jaundice \\
\hline & $\begin{array}{l}\text { Thitonia } \\
\text { diversifolia }\end{array}$ & $\mathrm{L}$ & $\mathrm{M}, \mathrm{i}, \mathrm{D}(\mathrm{W})$ & & $\begin{array}{l}\text { Wired, Scabies, antifilaria, Anti- } \\
\text { leukemic }\end{array}$ \\
\hline & $\begin{array}{l}\text { Vernonia } \\
\text { amygdalina }\end{array}$ & $\mathrm{L}$ & $\mathrm{M}, \mathrm{D}(\mathrm{W})$ & $08 / 308$ & $\begin{array}{l}\text { Gastric disorders, asthma, } \\
\text { stomach ache, wounds, } \\
\text { anthelmintic, malaria, wound } \\
\text { healing, headache }\end{array}$ \\
\hline Bromeliaceae & Ananas comosus & $\mathrm{F}$ & $\mathrm{M}, \mathrm{D}(\mathrm{W})$ & $07 / 308$ & malaria \\
\hline \multirow[t]{3}{*}{ Burseraceae } & $\begin{array}{l}\text { Canarium } \\
\text { schweinfurthii }\end{array}$ & SB & $\mathrm{D}(\mathrm{W})$ & $03 / 308$ & $/ /$ \\
\hline & $\begin{array}{l}\text { Commiphora } \\
\text { kerstingii }\end{array}$ & $\mathrm{L}, \mathrm{F}$ & $\mathrm{D}(\mathrm{W})$ & $02 / 308$ & Anti-helmentic and hypertension \\
\hline & Withania somnifera & $\mathrm{L}$ & $\mathrm{I}, \mathrm{D}(\mathrm{W})$ & $01 / 308$ & aphrodisiac \\
\hline \multirow[t]{3}{*}{ Caesalpiniaceae } & Delonix regia & $\mathrm{Fl}$ & $\mathrm{M}, \mathrm{I}(\mathrm{W})$ & $01 / 308$ & I \\
\hline & Senna alata & $\mathrm{L}$ & $\mathrm{I}, \mathrm{D}, \mathrm{M}(\mathrm{W})$ & $06 / 308$ & fever, stomach ache \\
\hline & Tamarindus indica & $\mathrm{L}, \mathrm{F}$ & $\mathrm{D}(\mathrm{W})$ & $05 / 308$ & Digestive disorders \\
\hline Caricaceae & $\begin{array}{l}\text { Carica papaya } \\
\text { Linn }\end{array}$ & $\begin{array}{l}\mathrm{L}, \mathrm{R}, \\
\text { sead }\end{array}$ & $\mathrm{M}, \mathrm{D}(\mathrm{W})$ & $17 / 308$ & $\begin{array}{l}\text { Jaundice, Dengue, Belly ache, } \\
\text { Hemorrhoid, Gastritis, Malaria, } \\
\text { Anti-helmentic, blood purification }\end{array}$ \\
\hline \multirow[t]{2}{*}{ Combretaceae } & $\begin{array}{l}\text { Combretum } \\
\text { micranthus }\end{array}$ & $\mathrm{L}, \mathrm{F}, \mathrm{SB}$ & $\mathrm{D}(\mathrm{W})$ & $04 / 308$ & I \\
\hline & $\begin{array}{l}\text { Terminalia } \\
\text { macroptera }\end{array}$ & SB & $\mathrm{D}(\mathrm{W})$ & $03 / 308$ & l \\
\hline Convolvulaceae & Ipomoea batatas $\mathrm{L}$. & $\mathrm{L}$ & $\mathrm{I}, \mathrm{D}(\mathrm{W})$ & $06 / 308$ & Against colon cancer \\
\hline
\end{tabular}




\begin{tabular}{|c|c|c|c|c|c|}
\hline \multirow[t]{3}{*}{ Euphorbiaceae } & $\begin{array}{l}\text { Euphorbia } \\
\text { prostrata }\end{array}$ & WP & $\mathrm{M}(\mathrm{W})$ & $03 / 308$ & dysentery \\
\hline & $\begin{array}{l}\text { Euphorbia hirta } \\
\text { Linn }\end{array}$ & WP & $\mathrm{M}(\mathrm{W})$ & $07 / 308$ & $\begin{array}{l}\text { Dysentery, Stomach ache, } \\
\text { Diarrhea, hemostatic, jaundice, } \\
\text { asthma }\end{array}$ \\
\hline & Manihotes culenta & $\mathrm{L}$ & $\mathrm{M}, \mathrm{I}(\mathrm{W})$ & $02 / 308$ & / \\
\hline \multirow[t]{11}{*}{ Fabaceae } & Acacia polyakanta & $\mathrm{Fl}$ & $\mathrm{D}(\mathrm{W})$ & $01 / 308$ & I \\
\hline & Erythrina $s p$ & SB & $\mathrm{D}(\mathrm{W})$ & $02 / 308$ & I \\
\hline & Daniella oliveri & SB & $\mathrm{D}(\mathrm{W})$ & $01 / 308$ & Malaria, rheumatism \\
\hline & Pakia biglobosa & $\mathrm{L}, \mathrm{F}$ & $\mathrm{D}, \mathrm{I}(\mathrm{W})$ & $01 / 308$ & I \\
\hline & $\begin{array}{l}\text { Piliostigma } \\
\text { thonningii }\end{array}$ & $\mathrm{L}, \mathrm{SB}$ & $\mathrm{B}, \mathrm{M}(\mathrm{W})$ & $11 / 308$ & Rheumatism, Fever, gastritis \\
\hline & Phaseolusvulgaris & $\mathrm{L}$ & $\mathrm{D}, \mathrm{M}(\mathrm{W})$ & $01 / 308$ & kidney infections \\
\hline & Senna occidentalis & $\mathrm{L}$ & $\mathrm{D}, \mathrm{M}(\mathrm{W})$ & $02 / 308$ & Malaria, pregnancy \\
\hline & $\begin{array}{l}\text { Senegalia } \\
\text { polyacantha }\end{array}$ & SB & $\mathrm{D}, \mathrm{I}(\mathrm{W})$ & $01 / 308$ & \\
\hline & $\begin{array}{l}\text { Desmodium } \\
\text { intortum }\end{array}$ & $\mathrm{L}, \mathrm{SB}$ & $\mathrm{D}(\mathrm{W})$ & $04 / 308$ & / insectifuge \\
\hline & Senna spactabilis & $\mathrm{L}$ & $\mathrm{D}(\mathrm{W})$ & $01 / 308$ & Sore throat \\
\hline & Senna tora & L, SB & $\mathrm{D}(\mathrm{W})$ & $01 / 308$ & Jaundice \\
\hline Hypericaceae & $\begin{array}{l}\text { Harungana } \\
\text { madagascariensis }\end{array}$ & $\mathrm{L}, \mathrm{SB}$ & $\mathrm{D}(\mathrm{W})$ & $11 / 308$ & $\begin{array}{l}\text { Liver dysfunction, stomach ache, } \\
\text { itching }\end{array}$ \\
\hline \multirow[t]{3}{*}{ Lamiaceae } & Thymus vulgarus/l & WP & $\mathrm{I}(\mathrm{W})$ & $01 / 308$ & I \\
\hline & Basilicum осітит & WP & $\mathrm{I}(\mathrm{W})$ & $01 / 308$ & l \\
\hline & Persea americana & $\mathrm{L}, \mathrm{SB}$ & $\mathrm{D}(\mathrm{W})$ & $20 / 308$ & $\begin{array}{l}\text { Amenorrhea, abortive, anemia, } \\
\text { bloating, weight lost, good } \\
\text { pregnancy, dysentery, diarrhea, } \\
\text { cough, fatigue, pain killer, } \\
\text { hemorrhoid, diarrhea, aphrodisiac, } \\
\text { wormer, hypertension, malaria, } \\
\text { abdominal pain }\end{array}$ \\
\hline Lauraceae & Laurusrobilis & $\mathrm{L}$ & $\mathrm{D}(\mathrm{W})$ & $01 / 308$ & \\
\hline \multirow[t]{2}{*}{ Liliaceae } & $\begin{array}{l}\text { Allium cepa } L . \\
\text { Aloe buttneri } L \text {. }\end{array}$ & $\mathrm{L}, \mathrm{B}$ & $\mathrm{M}, \mathrm{D}, \mathrm{I}(\mathrm{W})$ & $03 / 308$ & I \\
\hline & Allium sativum & $\mathrm{L}, \mathrm{B}$ & $\mathrm{D}, \mathrm{I}(\mathrm{W}, \mathrm{Ml})$ & $05 / 308$ & $\begin{array}{l}\text { Anticancer, antiviral, } \\
\text { hypertension, rheumatism }\end{array}$ \\
\hline Loganiaceae & Strychnos spirosa & $\mathrm{L}, \mathrm{SB}, \mathrm{R}$ & & $02 / 308$ & Digestive disorders \\
\hline Moringaceae & Moringaoleifera & $\mathrm{L}, \mathrm{F}$ & $\mathrm{M}, \mathrm{D}(\mathrm{W})$ & $04 / 308$ & $\begin{array}{l}\text { Blood purification, hypertension, } \\
\text { diabetes, malaria }\end{array}$ \\
\hline Moraceae & Ficus thonningii & SB & $\mathrm{D}(\mathrm{W})$ & & Cough, itchy skin \\
\hline Meliaceae & Azadirachta indica & $\mathrm{L}, \mathrm{SB}, \mathrm{F}$ & I, D (W H) & $02 / 308$ & malaria, diarrhea \\
\hline \multirow[t]{2}{*}{ Musaceae } & Musa sinensis & $\mathrm{L}$ & $\mathrm{D}(\mathrm{W})$ & $03 / 308$ & Wound \\
\hline & Musa paradisiaca & $\mathrm{L}$ & $\mathrm{D}(\mathrm{W})$ & $04 / 308$ & malaria \\
\hline Mimosoideae & Pakia biglobossa & $\mathrm{L}, \mathrm{F}$ & $\mathrm{I}, \mathrm{D}(\mathrm{W})$ & $01 / 308$ & Digestive disorders \\
\hline \multirow[t]{2}{*}{ Myrtaceae } & $\begin{array}{l}\text { Eucalyptus } \\
\text { globulus }\end{array}$ & $\mathrm{L}$ & $\mathrm{D}(\mathrm{W})$ & & $\begin{array}{l}\text { Asthma, oral hygiene, bronchitis, } \\
\text { cold, flu, malaria }\end{array}$ \\
\hline & Psidium guayava & $\mathrm{L}$ & M, D (W) & $17 / 308$ & $\begin{array}{l}\text { Cold, flu, angina, headache, } \\
\text { digestive disorders, stomach ache, } \\
\text { dermatitis, leucorrhea, diarrhea, }\end{array}$ \\
\hline
\end{tabular}


yellow fever, deworming, rheumatism

\begin{tabular}{|c|c|c|c|c|c|}
\hline Onagraceae & $\begin{array}{l}\text { Ludwigia } \\
\text { abyssinica }\end{array}$ & WP & $\mathrm{D}(\mathrm{W})$ & $01 / 308$ & Prostatitis \\
\hline Phyllanthaceae & $\begin{array}{l}\text { Hymenocardia } \\
\text { acida }\end{array}$ & $\mathrm{L}$ & $\mathrm{D}(\mathrm{W})$ & $01 / 308$ & Gastritis \\
\hline \multirow[t]{2}{*}{ Poaceae } & $\begin{array}{l}\text { Bambuseae } \\
\text { vulgarus }\end{array}$ & $\mathrm{L}$ & $\mathrm{D}(\mathrm{W})$ & $02 / 308$ & \\
\hline & Zea mays & $\mathrm{Fl}$ & $\mathrm{D}(\mathrm{W})$ & $02 / 308$ & Rheumatoid arthritis \\
\hline Rosaceae & Prunus domestica & $\mathrm{L}$ & $\mathrm{D}(\mathrm{W})$ & $01 / 308$ & \\
\hline Rhamnaceae & $\begin{array}{l}\text { Zizyphus } \\
\text { mauritiana }\end{array}$ & $\mathrm{L}$ & $\mathrm{D}$ & $01 / 308$ & Antidiarrheal \\
\hline rubiaceae & $\begin{array}{l}\text { Sarcocéphalus } \\
\text { latifolius }\end{array}$ & $\begin{array}{l}\mathrm{L}, \\
\text { SB,R }\end{array}$ & $\mathrm{D}$ & $01 / 308$ & \\
\hline \multirow[t]{4}{*}{ Rutaceae } & Citrus arantium & $\mathrm{L}, \mathrm{F}$ & $\mathrm{D}, \mathrm{M}(\mathrm{W})$ & $01 / 308$ & Rhume grippe \\
\hline & Citrus limon & $\mathrm{L}, \mathrm{F}$ & $\mathrm{D}, \mathrm{M}(\mathrm{W})$ & $11 / 308$ & $\begin{array}{l}\text { Cough, Flu, Cold, Headache, } \\
\text { Toning, Stomach Pain, } \\
\text { Rheumatism }\end{array}$ \\
\hline & Citrus reticulata & $\mathrm{L}$ & $\mathrm{D}, \mathrm{M}(\mathrm{W})$ & $01 / 308$ & Overweight \\
\hline & Citrus maxima & $\mathrm{L}$ & $\mathrm{D}, \mathrm{M}(\mathrm{W})$ & $01 / 308$ & $\begin{array}{l}\text { Heart problem, Facilitates the } \\
\text { expulsion of gases, colds }\end{array}$ \\
\hline Rhamnaceae & Ziziphys mauritana & $\mathrm{L}, \mathrm{SB}$ & $\mathrm{D}(\mathrm{W})$ & $01 / 308$ & Gonorhea, dysentery \\
\hline Solanaceae & Withania somnifera & $\mathrm{L}$ & $\mathrm{D}(\mathrm{W})$ & $01 / 308$ & \\
\hline Sapotaceae & Vitellaria paradoxa & $\mathrm{L}, \mathrm{SB}$ & $\mathrm{D}(\mathrm{W})$ & $06 / 308$ & Rheumatism \\
\hline \multirow[t]{2}{*}{ Solanaceae } & Solanum torvum & $\mathrm{L}, \mathrm{F}$ & $\mathrm{D}(\mathrm{W})$ & $05 / 308$ & I \\
\hline & $\begin{array}{l}\text { Solanum } \\
\text { lycopersicum }\end{array}$ & $\mathrm{L}, \mathrm{F}$ & $\mathrm{M}(\mathrm{W})$ & $01 / 308$ & Heart disease \\
\hline Tropaeolaceae & Tropaelummajus & WP & $\mathrm{D}(\mathrm{W})$ & $01 / 308$ & Headache \\
\hline Zingiberaceae & Zingiber officinale & $\mathrm{R}$ & $\mathrm{I}, \mathrm{M}(\mathrm{W})$ & $01 / 308$ & Good digestion \\
\hline
\end{tabular}

B: bark; D: decoction; F: fruit; FC: Frequency of citation, Fl: flower, infusion; L: leaves; M: maceration; R: roots; SB: stem Back; W: Water; WP: whole plant.

Table 3: Origin of the knowdledge, different types of antityphoid preparations according to study erea, way of administration, dose and duration of treatment.

\begin{tabular}{|c|c|c|c|c|}
\hline $\begin{array}{c}\text { Sub- } \\
\text { division }\end{array}$ & $\begin{array}{l}\text { Origine of knowledge } \\
\text { (number of } \\
\text { preparation) }\end{array}$ & Scientific name & $\begin{array}{l}\text { Solvent used, mode of } \\
\text { preparation and route of } \\
\text { administration }\end{array}$ & $\begin{array}{l}\text { Dose; } \\
\text { duration of } \\
\text { the treatment }\end{array}$ \\
\hline \multirow{3}{*}{  } & \multirow{3}{*}{ Ancestors (10) } & $\begin{array}{l}\text { Crysanthélium Africanium } \\
\text { Vernonia amygdalina } \\
\text { Persea americana }\end{array}$ & Water, Decoction, oral & $3 \mathrm{G} / \mathrm{D} ; 14 \mathrm{D}$ \\
\hline & & $\begin{array}{l}\text { Sarcocéphalus latifolius } \\
\text { Euphorbia prostrata } \\
\text { Bidenspilosa }\end{array}$ & Water, Maceration, oral & $3 \mathrm{G} / \mathrm{D} ; 14 \mathrm{D}$ \\
\hline & & $\begin{array}{l}\text { Persea americana } \\
\text { Spdium goyajava } \\
\text { Mangifora indica } \\
\text { Carica papaya } \\
\text { Annona senegalensis }\end{array}$ & Water, Decoction, oral & 4G/D ; 10D \\
\hline
\end{tabular}


Apiumgraveolens

Tithonia diversifolia

Canariumschweinfurthii

Mangiforaindica

\begin{tabular}{ll}
\hline Annona senegalensis & Water, Decoction, oral \\
Withania somnifera & \\
Persea americana & \\
Bambuseae vulgarus & \\
Harungana & \\
madagascariensis & \\
Terminalia macroptera & \\
Piliostigma thonningii & \\
Thymus vulgarus & \\
Eucaluptus globulus & \\
\hline Allium sativum & Water, Maceration, oral \\
Citrus limon & Water, Decoction, oral \\
\hline Ananas comosus & \\
Citrus limon & \\
Eucaluptus globulus & \\
Psidium guayava & \\
Harungana & \\
madagascariensis & \\
\hline Euphorbia hirta & \\
Mandiferaindica & \\
Annona senegalensis & \\
Bidens pilosa & \\
Strychnos spirosa & \\
Psidium guajava & \\
Harungana & \\
madagascariensis & \\
\hline Perseater, Decoction, oral \\
\end{tabular}

Persea americana

Water, Decoction, oral

3G/D ; 14D

Carica papaya

Thitonia diversifolia

Commiphora kerstingii

Citrus limon

Allium sativum

Moringa oleifera

Combretum micranthus/04 Water, Decoction, oral 4G/D, 21D

Annona senegalensis

Combretum micranthus

Sarcocéphalus latifolius

Water, Maceration, oral 3G/D, 14D

Euphorbia prostrata

Aloe vera

Carica papaya

Initiation (10)
Allium sativum

Sarcocephalus latifolius

Harungana

madagascariensis

Water, Maceration, oral 1G/D ; 14D
I

Water, Decoction, oral

3G/D ; 14D 
Vernonia conferata

Water, Maceration, oral

2G/D, 21D

Persia americana

Psidiumguayava

Water, Decoction, oral

1G/D ;14D

Musa sinensis

Persia americana

Carica papaya

Ananas comosus

\begin{tabular}{lll}
\hline $\begin{array}{l}\text { Harungana } \\
\text { madagascariensis }\end{array}$ & Water, Decoction, oral & 2G/D ;7D \\
\hline $\begin{array}{l}\text { Basilicum ocimum } \\
\text { Annona senegalensis } \\
\text { Senegalia polyacantha }\end{array}$ & Water, Decoction, oral & 3G/D ; 10D \\
Voacanga africana & & \\
$\begin{array}{l}\text { Combretum micranthum } \\
\text { Sarcocéphalus latifolius }\end{array}$ & & \\
\hline $\begin{array}{l}\text { Citrus limon } \\
\text { Psidium guayava }\end{array}$ & Water, Infusion, oral & 3G/D;7D \\
$\begin{array}{l}\text { Laurus robilis } \\
\text { Moringa oleifera }\end{array}$ & \\
\hline $\begin{array}{l}\text { Carica papaya } \\
\text { Bidens pilosa } \\
\text { Mandifera indica }\end{array}$ & & \\
\hline $\begin{array}{l}\text { Senna siamea } \\
\text { Persia americana } \\
\text { Vernonia amygdalina } \\
\text { Annona muricata }\end{array}$ & & \\
\hline
\end{tabular}

Prunus domestica

Water, Decoction, oral

2G/D ; 14D

Tamaridus indica

/Honey, Maceration, oral

Musa in

Tithoniadiversifolia/02

Strychnasspinosa

Senna alata

Lannea acida/7

Carica papaya

Water, Decoction, oral

3G/D ; 10D

Ananas comosus

Annonas senegalensis

\begin{tabular}{lll}
\hline $\begin{array}{l}\text { Ipomea batatas } \\
\text { Vitellaria paradoxa }\end{array}$ & $\begin{array}{l}\text { Water, Maceration, oral } \\
\text { honey/kernel/milk }\end{array}$ & 2TS/D $; 10 \mathrm{D}$ \\
\hline Musa spp & Water, Decoction, oral / & 2G/D ; 10D \\
Rock salt & Honey or kernel oil & 4TS/D ;10D
\end{tabular}

Psidum guayava

Maceration, oral

Persea americana

Ipomoeabatatas

Sarcocéphalus latifolius

$\begin{array}{ll}\text { Citrus limon } & \text { Water, Decoction, oral } \\ \text { Musa paradisiaca } & \\ \text { Persea americana } & \\ \text { Spidiumgoyajava } & \\ \text { Mandiferaindica } & \\ \text { Caricapapaya } & \end{array}$


Annonassenegalensis

Voacangaafricana

Citrus reticulata/01

Acacia polyakanta

ananas

\begin{tabular}{|c|c|c|}
\hline $\begin{array}{l}\text { Bidens pilosa } \\
\text { Eucalyptus globulus } \\
\text { Annonamuricata } 02 \\
\text { Senna occidentalis } \\
\text { Persea americana } \\
\text { Sarcocéphalus latifolius }\end{array}$ & Water, Decoction, oral & 3G/D ; 14D \\
\hline $\begin{array}{l}\text { Annona senegalensis } \\
\text { Pakia biglobosa } \\
\text { Euphorbia hirta } \\
\text { Senna siamea } \\
\text { Tamarindusindica } \\
\text { Harungana } \\
\text { madagascariensis }\end{array}$ & Water, Decoction, oral & $3 \mathrm{G} / \mathrm{D} ; 21 \mathrm{D}$ \\
\hline $\begin{array}{l}\text { Mandifera indica } \\
\text { Thitonia diversifolia } \\
\text { Apium gravedens } \\
\text { Euphorbia prostrata } \\
\text { Senna alata } \\
\end{array}$ & Water, Decoction, oral & 4G/D ; 14D \\
\hline $\begin{array}{l}\text { Tamarindus indica } \\
\text { Caricapapaya } \\
\text { Ananas comosus } \\
\text { Annona senegalensis } \\
\text { Solanum torvum } \\
\text { Mangiforaindica }\end{array}$ & Water, Decoction, oral & $3 \mathrm{G} / \mathrm{D} ; 21 \mathrm{D}$ \\
\hline $\begin{array}{l}\text { Allium sativum } \\
\text { Carica papaya } \\
\text { Persea americana } \\
\text { Citrus llmon } \\
\text { Psidium guajava } \\
\text { Harungana } \\
\text { madagascariensis }\end{array}$ & $\begin{array}{l}\text { Cow milk, } \\
\text { Maceration, oral }\end{array}$ & 3G/D ; 10D \\
\hline
\end{tabular}

Carica papaya

Water, Maceration, oral

3G/D ; 7D

Anona comosus

Initiation (10)

Annona senegalensis

\begin{tabular}{lll}
\hline $\begin{array}{l}\text { Lannea acida } \\
\text { Solanum torvum }\end{array}$ & Water, Decoction, oral & 3G/D ; 14D \\
\hline $\begin{array}{l}\text { Caricapapaya } \\
\text { Zea mays }\end{array}$ & Water, Decoction, oral & 6G/D ; 4D \\
$\begin{array}{l}\text { Citrus limon } \\
\text { Mandifora indica }\end{array}$ & & \\
$\begin{array}{l}\text { Delonix regia } \\
\text { Sarcocéphalus latifolius }\end{array}$ & & \\
\hline Allium sepa & Water, Maceration, oral & 2G/D ; 7D \\
\hline Pilostigma thoningii & Water, Decoction, oral & 2G/D ; 14D \\
\hline Mandifera indica & Water, Infusion, oral & 2G/D ; 14D
\end{tabular}


Caccia occidentalis

Ludwigia abyssinica

Canarium schweinfurthii

\begin{tabular}{|c|c|c|}
\hline $\begin{array}{l}\text { Persea americana } \\
\text { Allium cepa }\end{array}$ & Water, Decoction, oral & $3 \mathrm{G} / \mathrm{D} ; 14 \mathrm{D}$ \\
\hline Carica papaya & Water, Infusion, oral & 3G/D ; 7D \\
\hline $\begin{array}{l}\text { Vitellaria paradoxa } \\
\text { Mandiféra indica } \\
\text { Solanum torvum }\end{array}$ & Water, Decoction, oral & $3 \mathrm{G} / \mathrm{D} ; 14 \mathrm{D}$ \\
\hline $\begin{array}{l}\text { Persea americana } \\
\text { Psidium guajava } \\
\text { Musa paradisiaca } \\
\text { natron }\end{array}$ & Water, Decoction, oral & $3 \mathrm{G} / \mathrm{D} ; 10 \mathrm{D}$ \\
\hline Ipomoea battatos & Kernel oil, Maceration, oral & 4TS/D 10D \\
\hline Vitellaria paradoxa & $\begin{array}{l}\text { Cow Milk, Infusion, oral } \\
\text { milk }\end{array}$ & 4G/D ; 14D \\
\hline $\begin{array}{l}\text { Percea americana } \\
\text { Musa paradisiaca } \\
\text { Psidium guajava }\end{array}$ & Water, Decoction, oral & 2G/D ; 14D \\
\hline Ipomoea batatas & $\begin{array}{l}\text { Azadirachta india oil, } \\
\text { Maceration, oral }\end{array}$ & 4TS/D ; 7D \\
\hline Vitellaria paradoxa & Water, decoction, oral & 3G/D ; 14D \\
\hline $\begin{array}{l}\text { Zizyphus mauritiana } \\
\text { Hurungana } \\
\text { madagascariensis }\end{array}$ & Water, Decoction, oral & $2 \mathrm{G} / \mathrm{D} ; 14 \mathrm{D}$ \\
\hline
\end{tabular}

Citrus limen Water, Decoction, oral 3G/D;14D

Senna spactabilis

Allium cepa

Crhysanthellum africanium

Sena tora

Senna alata

Annona senegalensis

Pakia biglobossa

Eucalyptus globulus

MBE

Ancestor

Harungana

(14)

madagascariensis

Manihotes culenta

Ipomoea batatas

Senna alata

\begin{tabular}{lll}
\hline $\begin{array}{l}\text { Zea mais } \\
\text { Citrus avrantium }\end{array}$ & Cowmilk, Maceration, oral & 2G/D ; 14D \\
\hline $\begin{array}{l}\text { Solanum lycopersicum } \\
\text { Zingiber offocinale }\end{array}$ & Water, Decoction, oral & 2-4G/D ; 10D \\
$\begin{array}{l}\text { Phasealus vulgaris } \\
\text { Lannea acida }\end{array}$ & Water, Decoction, oral & \\
Erythrina senegalensis & & \\
\hline $\begin{array}{l}\text { Annona senegalensis } \\
\text { Withania somnifera }\end{array}$ & Water, Decoction, oral & 2G/D ; 14D \\
$\begin{array}{l}\text { Persea americana } \\
\text { Bambuseae vulgarus }\end{array}$ & & \\
\end{tabular}


Harungana

madagascariensis

Terminalia macroptera

Piliostigma thonningii

Citrus limon

eucaluptusglobulus

Psidium guajava

Psidium guajava $\quad$ Water, Maceration, oral 2G/D ; 14D

Vernonia amygdalina

Annona senegalensis

Pilostigma tanningii

Ehphorbia hirta Water, Maceration, oral 2G/D ;10D

Persea améridana

Carica papaya

Gympobogannadus

Pilostigma tanningii

Water, Maceration, oral

2G/D ; 14D

Annona senegalensis

Psidium guayava

Persea americana

Mandiféra indica

Mangifera indica

Water, Decoction, oral

2G/D ; 10D

Thitonia diversifolia

Persea americana

Carica papaya

Piliostigma thonningit

Psidium guayava

Moringa oleifera

Vernonia amygdalina

Piliostigma thonningii

Eucalyptus camaldulensis

Psidium guayava

Annona senegalensis

\begin{tabular}{lll}
\hline Citrus sinensis & Azaderachta indica oil, & 2G/D ; 7D \\
Azadiracta indica & Maceration, oral
\end{tabular}

Citrus maxima

Sarcocéphalus latifolius

Carica papaya

Water, Infusion, oral

2G/D ; 14D

Psidum guayava

Musa spp

eucalyptus globulus

Combretum micranthum

Initiation (06)

\begin{tabular}{lll} 
Lannea acida & & \\
\hline Euphorbia hirta & $\begin{array}{l}\text { Water, Maceration, oral } \\
\text { / Water, Infusion, oral }\end{array}$ & 2G/D ; 10D \\
\hline Citrus sinensis & Water, Decoction, oral & 2G/D ; 10D \\
Psidum guayava & & \\
Zizyphys mauritana & & \\
\hline Vernonia amygdalina & Water, Decoction, oral & \\
Eucalyptus globulus & & \\
Annonasenegalensis & &
\end{tabular}


Crhysanthellum africanium

Solanum torvum

\begin{tabular}{ll}
\hline Harungana & Water, Decoction, oral \\
madagascariensis & \\
Terminalia macroptera & \\
Piliostigma thonningii & \\
Caricapapaya & \\
Sarcocéphalus latifolius & \\
Vitellaria paradoxa & \\
Solanum torvum &
\end{tabular}

Tropoelummajus $\quad$ Water, Decoction, oral 3G/D ; 7D

Moringa oleifera

Psidium guajava

Carica papaya

Citrus lemon

Lannea acida

Erythrina

Vernonia amygdalina

Water, Maceration, oral

2G/D ; 14D

Aloes vera /Water, Decoction, oral

Persea americana

Citrus limon

Euphorbia hirta

\begin{tabular}{lll}
\hline Lannea acida & Water, Maceration, oral & 3G/D ; 10D \\
Crhysanthellum africanium & / Water Decoction, oral & \\
\hline Persea americana & Water, Maceration, oral & 3G/D ; 14D
\end{tabular}

Kommiphora kersthengii

Vernonia amydalina

Piliastigma thonningii

Citrus limon

Hymennocardia acida

Water, Maceration, oral

2G/D ; 10D

Psidium guajava

Daniella oliveri

Initiation and/or

Piliastigma thonningii

ancestors (12)

Ficus thonningii

Euphorbia hirta

Annona senegalensis

Water, Decoction, oral

3G/D ; 14D

Vitellaria paradoxa

Eucalyptus globulus

Aloe vera

Water, Maceration, oral

3G/D ; 4D

Azadirachta indica

Tamarindus indica

\begin{tabular}{ll}
\hline Lannea acida & Water, Decoction, oral \\
Sarcocéphalus latifolius & \\
Canarium schweinfurthii & \\
Erythrina senegalensis & \\
Senna alata &
\end{tabular}

Senna alata

\begin{tabular}{lll}
\hline Allium sativum & Milk, Infusion, oral & 2G/D ; 10D \\
\hline Citrus limon & Water, Decoction, oral / & 2G/D ; 14D \\
Eucalyptus globulus & Water, Infusion, oral &
\end{tabular}

Ananas comosus 
S. P. F. CHEGAING et al. / Int. J. Biol. Chem. Sci. 14(9): 3078-3096, 2020

\begin{tabular}{llc} 
Euphorbia hirta & & \\
\hline $\begin{array}{l}\text { Mandiféra indica } \\
\text { Citrus limon }\end{array}$ & Water, Decoction, oral & 2G/D ; 14D \\
Eucalyptus globulus & & \\
\hline $\begin{array}{l}\text { Pilostigma thomingii } \\
\text { Tamarindus indica }\end{array}$ & Water, Maceration, oral & $3 \mathrm{G} / \mathrm{D} ; 14 \mathrm{D}$ \\
Citrus limon & & \\
\hline $\begin{array}{l}\text { Senna alata } \\
\text { Manihotes culenta }\end{array}$ & Water, Maceration, oral & $3 \mathrm{G} / \mathrm{D} ; 10 \mathrm{D}$ \\
$\begin{array}{l}\text { Ipomea batatas } \\
\text { Bidens pilosa }\end{array}$ & \\
& & \\
& & \\
\end{tabular}

G/D, glass per day.

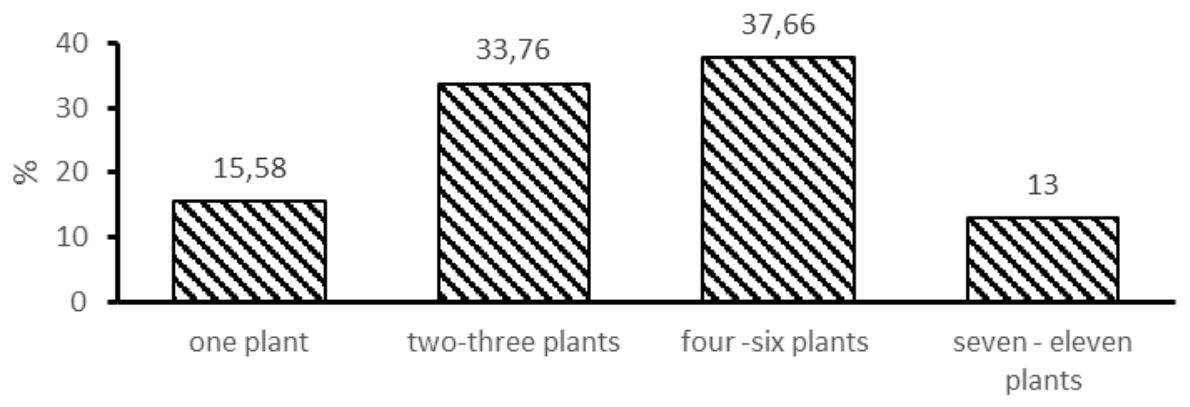

Figure 2: Number of plants per preparation.

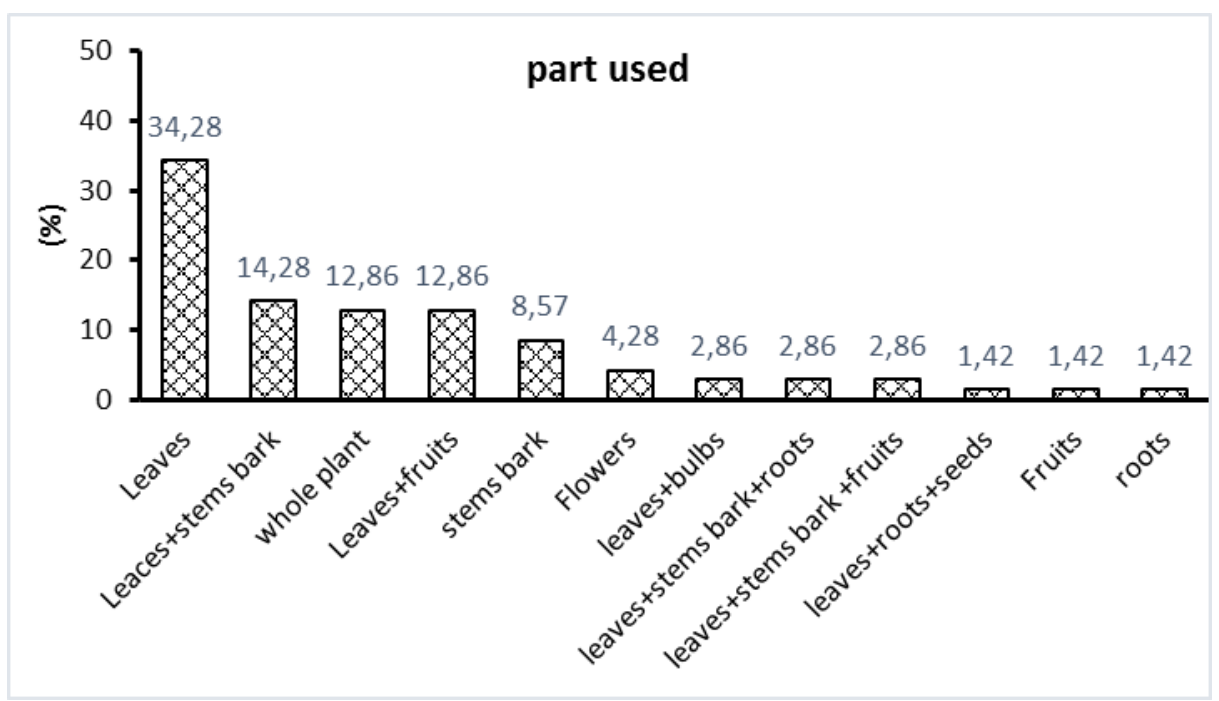

Figure 3: Parts of plant used. 


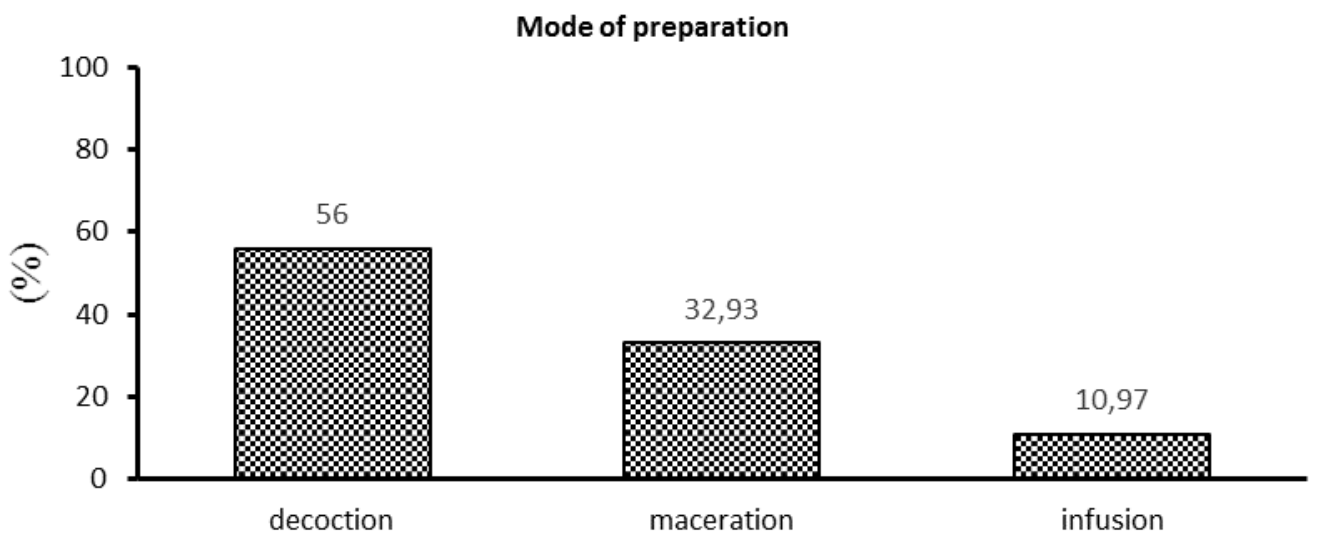

Figure 4: Modes of preparation.

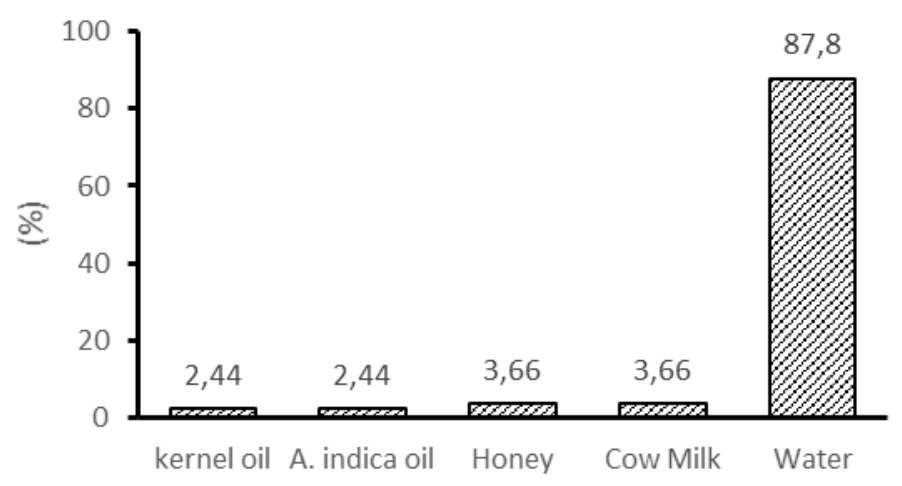

Figure 5: Solvents used for the preparations.

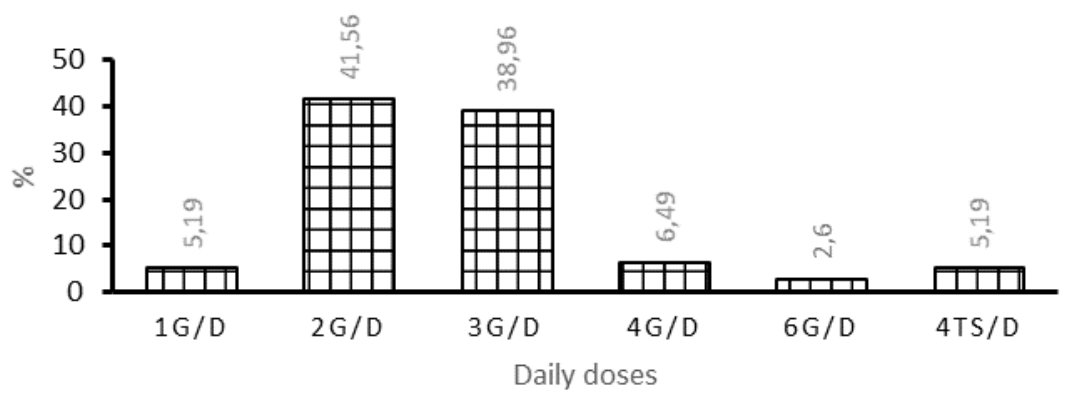




\section{DISCUSSION}

The high frequency of man participating in this study may be justified by the fact that the ancestors were initiating mainly their child boy to the practice of the traditional medicine. In addition, sociocultural habits of peoples in the northern part of Cameroon in general and in the study area in general limits the contacts between man and woman (Mokam, 2012). The precariousness in which the populations of the study area live could be the main reason why young people introduce themselves to the practice of traditional medicine. This is not only in order to provide for their family's needs, but also to improve the health of their population who, for most of them, are not able to get rid of pharmaceutical drugs.

The high frequency of Fabaceae (Table 2) may either be due to the wide range of biologically active compounds present in that family, but also because this family is the most widespread in the plant kingdom (Thomas et al., 2009). The high frequency of citation Persea americana (20/308) Annona senegalensis 17/308; Carica papaya 17/308; Psidium guayava (17/308) Citrus limon (11/308): Mangifera indica (14/308); Piliostigma thonningii (11/308) and Harungana madagascariensis (11/308) may be linked to their efficacy. Indeed, some of these plants have been reported worldwide to be used in the treatment of many diseases (Kengni et al., 2014: Yemele et al., 2015). Relatively to bacterial infection, the antibacterial activity of many of the above mentioned plants has been proved (Sospeter et al., 2015 ; Kengni et al., 2014; Amit, 2017 ; Amado et al., 2019 ; Srividya et Rama, 2019).

With a percentage of $34.28 \%$, leaves were the most plant parts used. In fact, leaves are known to accumulate plants secondary metabolites, which are known to possess many biological properties (OMS 2004; Focho et al., 2009a). Moreover, utilization of leaves and stem bark rather than root is advantageous for the survival of plants since their harvest does not induce the irreversible destruction of plants (Telefo et al., 2012). Besides, leaves are easier to harvest than root.
Recipes prepared with more than one medicinal plant is a common practice in our traditional medicine milieu and could be justified by the fact that, association of many plants and thus many secondary metabolites may improve the synergistic or additive effects of their constituents (Igoli et al., 2005). Apart from typhoid fever, these plants are used to cure several other complaints (Table 2). This could be attributed to large distribution of secondary metabolite with different properties in a single plant extract.

\section{Conclusion}

This study has shown that rural populations of the 3 targeted localities possess traditional knowledge on anti-infectious plants that are efficient and cost-effective for the treatment of typhoid fever. Nevertheless, these practices deserve to be validated experimentally in order to be considered as sustainable tools for the management of typhoid and para-typhoid fevers.

\section{COMPETING INTERESTS}

The authors declare that they have no competing interests.

\section{ACKNOWLEDGMENTS}

The authors are very grateful to the traditional healers for providing information about their practices.

\section{REFERENCES}

Agwu E, Ihongbe JC, Okogun GR, Inyang NJ. 2009. High incidence of co-infection with

malaria and typhoid in febrile HIV infected and aids patients in Ekpoma, Edo State, Nigeria. Brazilian Journal of Microbiology, 40: 329-32. DOI: 10.1590/S1517-83822009000200022

Amado DAV, Helmann GAB, Detoni AM, Carvalho SLC, Aguiar CM, Martin CA, Tiuman TS, Cottica SM. 2019. Antioxidant and antibacterial activity and preliminary toxicity analysis of four varieties of avocado (Persea Americana Mill.). Brazilian Journal of Food 
Technology, $22 . \quad$ DOI : https://doi.org/10.1590/1981-6723.04418

Amit G, Samrat SP, Pendharkar N. 2017. Antimicrobial and anti-inflammatory activity of aqueous extract of Carica papaya. Journal of Herbmed Pharmacology, 6(4): 148-152.

Annuaire statistique du Cameroun. Recueil des séries d'informations statistiques sur les activités économiques, sociales, politiques et culturelles du pays jusqu'en 2013.

Crump JA, Luby SP, Mintz ED. The global burden of typhoid fever. 2004. Bull. World Health, 82: 346-35. DOI: 10.1590/S0042-96862004000500008

David M. 2012. Les chemins d'émancipation de la femme sahélienne camerounaise. Nigerian Journal of the Humanities, 18: 61-81.

https://www.researchgate.net/publication $-/ 311545253$

Deciga-Campos M, Rivero-Cry I, ArriagaAlba M, Castaneda-Corral G, Angeles Lopez GE, Navarrete A, Mata R. 2007. Acute toxicity and mutagenic activity of Mexican plants used in traditional medicine. Journal of Ethnopharmacology, 110: 334-337. DOI: 10.1016/j.jep.2006.10.001

Etou AW, Ossibi MA, Elouma N, Epa C, Wossolo LBS, Bonose MN, Okemy AJM, Moutsambote, Abena AA. 2019. Chemical composition and diuretic potential of the essential oil of Cymbopogon densiflorus (Steud.) Stapf. (Poaceae) in the mouse. Int. J. Biol. Chem. Sci., 13(6): 2777-2784. DOI: 10.4314/ijbcs.v13i6.28

Kengni F, Fodouop SPC, Tala DS, Djimeli MN, Fokunang C, Gatsing D. 2016. Antityphoid properties and toxicity evaluation of Harungana madagascariensis Lam (Hypericaceae) aqueous leaf extract. Journal of Ethnopharmacology, 179: 137-145. DOI: 10.1016/j.jep.2015.12.037

Focho DA, Ndam WT, Fonge BA. 2009a. Medicinal plants of Aguambu Bamumbu in the Lebialem highlands, southwest province of Cameroon. African Journal of Pharmacy and Pharmacology, 3(1): 1-13. DOI : https://doi.org/10.5897/AJPP.9000204

Fodouop CSP, Gatsing D, Tagne SR, Tala SD, Tchoumboué J, Kuiate J-R. 2015. Effect of Salmonella Typhimurium infection on rat's cell oxidation and in vivo antioxidant activity of Vitellaria paradoxa and Ludwigia abyssinica aqueous extract. Asian Pacific Journal of Tropical Disease, 5(1): 38-46. DOI: 10.1016/S2222-1808(14)60624-1

Gatsing D, Nkeugouapi CFN, Nji-Nkah BF, Kuiate JR, Tchouanguep FM. 2010. Antibacterial activity, bioavailability and acute toxicity evaluation the leaf extract of Alchornea cordifolia (Euphorbiaceae). Int. J. Pharmacol., 6:173-82. DOI: 10.3923/ijp.2010.173.182

Lamy LGM, Ibrahima A, Ndjonka D, Mapongmetsem P-M. 2018. Etude ethnobotanique des sous-variétés de Syzygium guineense (Will.) DC. var. macrocarpum (Engl.) F. White dans les Hautes Savanes Guinéennes (Adamaoua, Cameroun). Int. J. Biol. Chem. Sci., 12(4): $\quad 1636-1649 . \quad$ DOI: 10.4314/ijbcs.v12i4.11]

Habte ET, Getachew F, Anteneh A. 2018. Typhoid fever: clinical presentation and associated factors in febrile patients visiting Shashemene Referral Hospital, southern Ethiopia Limenih. BMC Res. Notes, 11: 605. DOI : 10.1186/s13104018-3713-y

Igoli JO, Ogali OG, Tor-Anyiin, TA, Igoli NP. 2005. Traditional medicine practice amongst the Igede people of Nigeria. Part II. African Journal Traditional Complementary and Alternative Medicines, 2(2): 134-152. DOI: 10.4314/ajtcam.v2i2.31112

Lakshmi V, Ashok R, Susmita J, Shailaja VV. 2006. Changing trends in the antibiograms of Salmonella isolates at a tertiary care hospital in Hyderabad. Ind. J. Med. Microbio., 24(1): 45-48. DOI: 10.4103/0255-0857.19894 
Lunga LK, Tamokou JD, Fodouop CSP, Kuiate J-R, Tchoumboue J, Gatsing D. 2014a. Antityphoid and radical scavenging properties of the methanol extracts and compounds from the aerial part of Paullinia pinnata. SpringerPlus, 3(302) : 1-9. DOI: 10.1186/2193-18013-302

Madhulika U, Harish BN, Parija SC. 2004. Current pattern in antimicrobial susceptibility of Salmonella Typhi isolates in Pondicherry. Ind J. Med Res., 120: 111-114. DOI: 10.15413/ajmr.2013.0006

Mapongmetsem PM, Hamawa Y, Baye N, Froumsia C, Zigro M, Meiga OSL. 2009. Conservation et valorisation de la biodiversité dans les agrofôrets de case de la zone Soudano-Guinéenne. In Systematics and conservation of African Plants, van der Burgt X, van der Maesen J, Onana J-M (eds). Royal Botanic Gardens: Kew; 375-384.

Mapongmetsem PM, Kapchie VN, Tefempa BH. 2012. Diversity of local fruit trees and their contribution in sustaining the rural livelihood in the northern Cameroon. Ethiopian Journal of Environmental Studies and Management, 5(1): 32-46. DOI: 10.4314/ejesm.v5i1.5

Mfopa AN, Mbouna CDJ, Tchokouaha LRY, Tchuente MAT, Kouipou RMT, Fokou PVT, Kemgne EAM, Kamkumo RG, Boyom FF. 2017. In vitro and in vivo antiplasmodial activity of extracts from Polyalthia suaveolens, Uvaria angolensis

and Monodora tenuifolia (Annonaceae). Int. J. Biol. Chem. Sci., 11(1): 118-130. DOI: $10.4314 /$ ijbcs.v11i1.10.

Bamba M, Neut C, Bordage S, Dramane S, Kouadio NJ, Yacouba S, Samaillie J, Zamble A, Tah T, Tra-Bi-Fezan H, Sevser S. 2020. Screening phytochimique des extraits méthanoliques des feuilles de Combretum collinum et des racines de Anogeisus leiocarpus et effet antibactérien in vitro sur des souches multirésistantes de Staphylococcus aureus. Int. J. Biol. Chem. Sci.,14(6): 2362-2372.

DOI :

10.4314/ijbcs.v14i6.34]

Njolle AB, Tientche B, Asaah S, Forfuet DF, Kamga HLF. 2020. The Prevalence of Salmonellosis in Patients with Malaria Attending an Urban Hospital in Douala, Littoral Region, Cameroon. Journal of Advances in Medicine and Medical Research, 32(2): 32-45. DOI : doi.org/10.9734/jammr/2020/v32i23036 4

Sokoudjou JB, Chegaing Fodouop SP, Djoueudam FG, Kodjio N, Kana JR, Fowa AB, Kamsu TG, Gatsing D. 2019. Antisalmonellal and antioxidant potential of hydroethanolic extract of Canarium schweinfurthii Engl. (Burseraceae) in Salmonella enterica serovar Typhimurium-infected chicks. Asian Pac J. Trop. Biomed., 9(11): 474-483. DOI: 10.4103/2221-1691.270980

Sokoudjou JB, Njateng GSS, Fodouop CSP, Kodjio N, Atsafack SS, Fowa AB, Merline Namekong Djimeli NM, Gatsing D. 2018. In vitro antisalmonellal and antioxidant activities of Canarium schweinfurthii stem bark extracts. Academia Journal of Medicinal Plants, 6(10): $\quad 331-341 . \quad$ DOI: 10.15413/ajmp.2018.0166

Sospeter NN, Meshack AO, Silas MN, Samwel NO, John MN, Paul KK. 2015. Antituberculous, antimicrobial, cytotoxicity and phytochemical activity study of Piliostigma thonningii extract fractions. Journal of Medicinal Plants Research, 9(22): 655-663. DOI: 10.5897/jmpr2015.5822.

Srividya L, Rama A, Narsimha R. 2019. Antibacterial Activity of Carica papaya Leaves and Allium sativum Cloves Alone and in Combination against Multiple Strains. Pharmacogn J., 11(3): 600-602. DOI:10.5530/pj.2019.11.95.

Steele AD, Burgess DCH, Diaz Z, Carey ME, Zaidi AKM. 2016. Challenges and opportunities for typhoid fever control: A call for coordinated action. Clinical 
Infectious Diseases, 62: S4-8. DOI: $10.1093 / \mathrm{cid} / \mathrm{civ} 976$

Stuart D. 2004. Dangerous Garden: The Quest for Plants to Change Our Lives. Havard University Press, Cambridge.

Tala SD, Fodouop CSP, Tsafack ND, Kodjio N, Fokunang C, Gatsing D. 2018. Toxicological Investigations of Ethanolic Leaves Extract of Dissothis thollonii (Melastomataceae). Journal of Pharmaceutical Research International, 24(4): 1-14.

Tchobsala MM. 2013. Characterization and impact of wood logging on plant formations in Ngaoundéré District, Adamawa Region, Cameroon. Journal of Ecology and the Nature Environment, 5(10): 265-277. DOI: 10.5897/JENE10.102.

Telefo PB, Lemfack MC, Bayala B, Lienou LL, Goka CS, Yemele MD, Mouokeu C, Tagne SR, Moundipa FP. 2012. Enquête ethnopharmacologique des plantes utilisées dans le traitement de l'infertilité féminine dans les localités de Fossong-Wentcheng et Foto, Cameroun. Phytotherapie, 10(1): 25-34. DOI :10.1007/s10298-011-0678-6
Thomas E, Vandebroek I, Sanca S, Van Damme P. 2009. Cultural significance of medicinal plant families and species among the Quechua farmers in Apillapampa, Bolivia. Journal of Ethnopharmacology, 112: 60-67. DOI: 10.1016/j.jep.2008.11.021

WHO Weekly epidemiological record. 2014. Typhoid fever surveillance and vaccine use, South-East Asia and Western Pacific Regions, 2009-2013. WHO, 89: 429-440.

Yemele MD, Telefo PB, Lienou LL, Tagne SR, Fodouop CSP, Goka CS, Lemfack MC, Moundipa FP. 2015. Ethnobotanical survey of medicinal plants used for pregnant women's health conditions in Menoua division-West Cameroon. Journal of Ethnopharmacology, 160: 14-31. DOI: 10.1016/j.jep.2014.11.017

Zava DT, Dollbaum CM, Blen M. 1998. Estrogen and progestin bioactivity of foods, herbs and spices. Society for Experimental Biology and Medicine, 217: 369-371. DOI: 10.3181/00379727217-44247. 